\title{
Image Haze Removal Using Dark Channel Prior and Inverse Image
}

\author{
Lei Shi ${ }^{1}$, Xiao Cui ${ }^{1}$, Li Yang ${ }^{1}$, Zhigang Gai ${ }^{1}$, Shibo Chu ${ }^{1}$ and Jing Shi ${ }^{2}$ \\ ${ }^{1}$ Institute of Oceanographic Instrumentation, Shandong Academy of Sciences, Qingdao, China \\ ${ }^{2}$ Luoyang Institute of electro-optical devices, China Aviation Industry Corporation, Luoyang, China
}

\begin{abstract}
Image haze removal using dark channel prior is prone to encountering color distortion in sky and brightness region. To solve the problem, we proposed an improved method based on inverse image and dark channel prior. Firstly, we applied inverse image to estimating a new transmission map. The new transmission map can be used to modify original transmission map in order to avoid color distortion. Next, fast guider filter was applied to refining transmission map. Using the transmission map, we can recover a high quality haze-free image. Experimental results showed that the proposed method is feasible, and visibility can be enhanced.
\end{abstract}

\section{Introduction}

The contrast and visibility of the haze images are all decreased, which directly affects the air, water and road transport. Thus, simple and effective image haze removal algorithm is of great value to improve the robustness of the visual system.

In order to remove haze effect, many methods required additional information about the scene, such as multiple images captured in different weather conditions [1] or polarization angles [2]. Also, Kopf et al. [3] employed the depth knowledge of known 3D models for haze removal.

Recently, single image haze removal has made great progress. Many single image haze removal algorithms have been developed. Tan et al. [4] maximized the contrast of a hazy image, assuming that the haze-free image has a higher contrast than the hazy image. The results are visually compelling but may not be physically valid. Fattal et al. [5] decomposed the scene radiance of an image into the albedo and the shading, and then estimated the scene radiance, assuming that the shading and the object depth are locally uncorrelated. It can remove haze locally but cannot restore densely hazy images. Kim et al. [6] proposed a cost function produced by the contrast cost and the information loss cost. By optimizing the cost function, the proposed algorithm can enhance the contrast and preserve the information optimally.

He et al. [7] proposed a single image haze removal based on Dark Channel Prior (DCP). He randomly selected about 5000 images for DCP. The algorithm produced impressive result; however, the method may suffer from a limitation. It is that color distortion will appear in bright region (such as sky region). In this paper, we proposed an improved method based on [7] to deal with this case.

\section{Single image haze removal using dark channel prior}

The observed brightness of a hazy image can be modeled based on the atmospheric optics [7] via

$$
I(x)=J(x) t(x)+A(1-t(x))
$$

where, $I(x)$ is the observed haze image, $J(x)$ is the scene radiance(Haze-free Image), $A$ is the global atmospheric light, and $t(x) \in[0,1]$ is the medium transmission describing the portion of the light that is not scattered and reaches the camera.

He et al. [7] found that the minimum intensity in the non-sky patches on haze-free outdoor images should have a very low value, which is called dark channel prior(DCP). His observation told us that except for the sky region, the intensity of dark channel image $J_{\text {dark }}$ is low and tends to be zero. According to the DCP, the transmission can be estimated simply by:

$$
t_{f}(x)=1-\min _{c}\left(\min _{y \in \Omega(x)}\left(\frac{I_{c}(y)}{A^{c}}\right)\right)
$$

A constant parameter $\omega(0<\omega<1)$ was introduced into Equation (2) to keep a small amount of haze for the distant objects:

$$
t_{f}(x)=1-\omega \min _{c}\left(\min _{y \in \Omega(x)}\left(\frac{I_{c}(y)}{A^{c}}\right)\right)
$$

In the next step, He et al. [7] refined the transmission map using soft matting method [8]. For reducing the computational complexity, soon he applied guided image filter [9] to refine the transmission map. After the transmission map is estimated, the scene radiance $J(x)$ can be recovered according to Equation (1). The 
transmission $t_{f}(x)$ is restricted by a low bound to, which means that a small amount of haze is preserved in very dense haze regions. to is set as 0.1 . The final scene radiance $J(x)$ is recovered by

$$
J(x)=(I(x)-A) / t(x)+A, \quad t_{f}(x)=\max \left(t_{f}(x), t o\right)
$$

In [7], He analyzed about 5000 out-door images. Then, He told us that about $75 \%$ of the pixels in the dark channels have zero values, and the intensities of $90 \%$ of the pixels are below 25. This statistic gave very strong support to DCP. The algorithm can perform very well in most cases. Most pixels satisfied DCP. But, for bright pixels such as pixels in sky region, the method can not perform very well because these pixels cannot satisfy DCP. We presented an improved method to solve the problem.

\section{Image haze removal based on dark channel prior and inverse Image}

If there are large-area sky region and brightness object in out-door image, color distortion may appear in these regions when He's method [7] is applied, as described in Figure 2 under section 4. According to our observations, these regions will be brighter than others in most cases, and these differences between RGB channels values are very small. In general, the pixel values of RGB channels may be close to atmospheric light value $A$. DCP is not established in these regions. According to Equation (1), we obtained:

$$
t_{\text {actual }}(x)=\frac{1-\min _{c}\left(\min _{y \in \Omega(x)}\left(I_{c}(y) / A\right)\right)}{1-\min _{c}\left(\min _{y \in \Omega(x)}\left(J^{c}(y) / A\right)\right)}
$$

where, $t_{\text {actual }}(x)$ donates actual transmission value.

According to Equation (5), if $\operatorname{Ic}(y)$ value is close to atmospheric light value $A$, the term $\min _{c}\left(\min _{y \in \Omega(x)}\left(J^{c}(y) / A\right)\right)$ is not equal to 0.0 but close to 1.0, and $1-\min _{c}\left(\min _{y \in \Omega(x)}\left(J^{c}(y) / A\right)\right)$ is close to 0.0 . Therefore, $t_{\text {actual }}(x)$ is far above $t_{f}(x)$ based on DCP. The calculated transmission $t_{f}(x)$ value by Equation (3) is very small, and $J(x)$ value by Equation (4) is not accurate.

If $t o=0.1$ is chosen, $\max \left(t_{f}(x)\right.$, to $)$ may be also very small. If $I_{\mathrm{c}}(x)$ value is small to a certain extent, the absolute value of $(I(x)-A) / \max (t f(x)$, to $)$ will probably be amplified to a considerable value. At this time, $J(x)$ value will be very small in this channel. If other two RGB channel values are bigger than $A$, color distortion will appear.

Jiang et al. [11] added a controllable parameter $K$ in in order to adjust the transmission value of each pixel .When $|I(x)-A|$ is smaller than $K$, the pixel may belong to brightness region(such as sky). At the time, He will multiply the transmission value value $t f(x)$ by $\max (K /|I(x)-A|, 1)$. The value of $K$ is suggested to be 50 by Jiang. When $|I(x)-A|$ is bigger than $K$, the transmission value is $\max \left(t_{f}(x)\right.$, to $)$. Sun et al. [12] adjusted the transmission values based on $|I(x)-A|$. Wang et al. [13] applied sky segmentation based on gradient threshold, then set the transmission values in sky region directly into a fixed value. Deng et al. [14] separated firstly sky region from background image, then applied histogram equalization. Kim et al. [6] enhanced visibility and contrast by minimizing energy function. to was equal to 0.3 . All of the above algorithms can eliminate color distortion in some cases. These algorithms both depend on the experience value. But, there are also some situation is that they can't deal with it. Besides that, if we have to separating sky region from background image, this will result in burdensome calculation.

In [15], Ancuti et al. applied semi-inverse image to image haze removal. The semi-inverse image can be obtained by replacing the RGB values of each pixel on a per channel basis by the maximum of the initial channel value and its inverse. In many cases, the methods can do it well. In [6], Kim built cost function by two constraints, as described in Equation (6) and Equation (7):

$$
\begin{gathered}
t_{f}(x) \geq \min _{c}\left(\min _{y \in I(x)}\left(1-\frac{I_{c}(y)}{A}\right)\right) \\
t_{f}(x) \geq \max _{c}\left(\max _{y \in I(x)}\left(1-\frac{255-I_{c}(y)}{255-A}\right)\right)
\end{gathered}
$$

Equation (7) denoted inverse image can be used to haze removal. Kim's cost function included two portions. Besides Equation (6) and Equation (7), he also added the information loss cost. In [16], Tang et al. proposed similar constraints to [15].

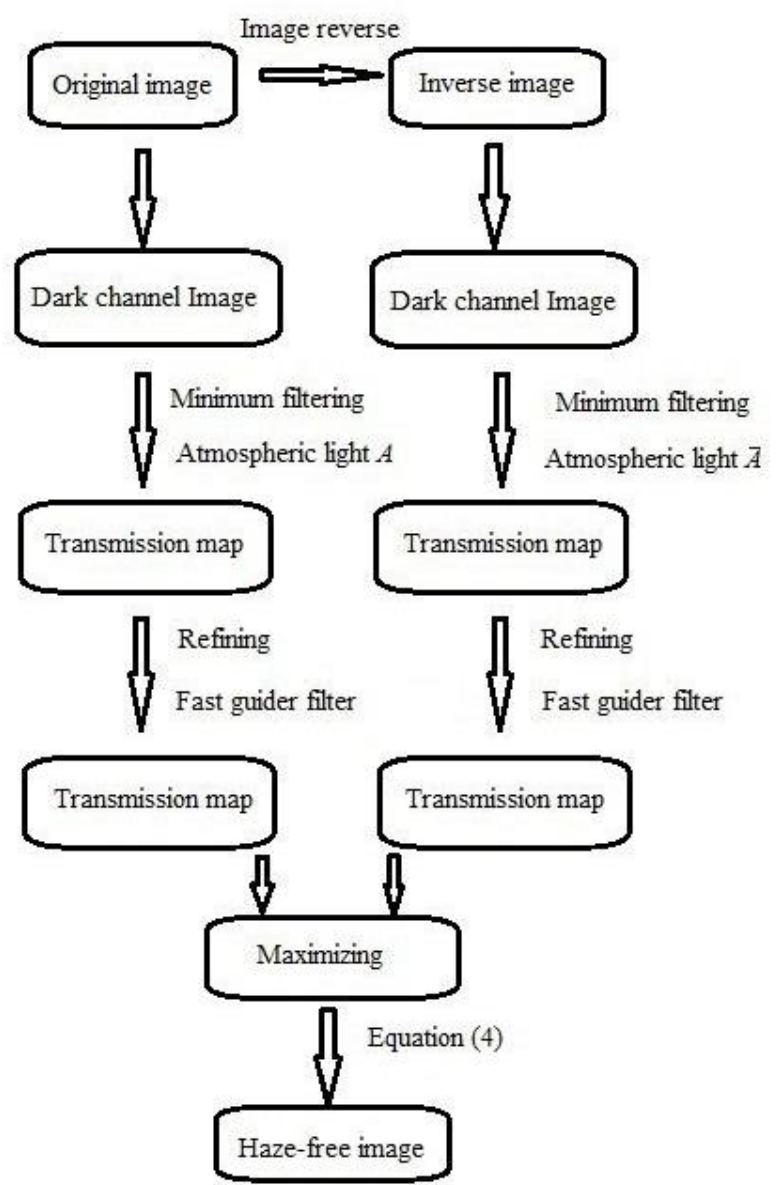

Figure 1. The flow chart. 
The three works [6], [15]-[16] told us that inverse image is very useful for haze removal. But, only it can not deal with all cases. We presented an improved method to overcoming color distortion using inverse image. The algorithm can be described as follows:

1) Calculated inverse image of original image, then calculated their dark channel images, and applied minimum filtering.

2) Calculated global atmospheric light value respectively.

3) Calculated initial transmission map respectively.

4) In order to reduce the complexity of calculation, we refined the two transmission map by fast guide filter [10] respectively.

5) Determined the maximum between the two refined transmission maps.

6) Recovered free-haze image by Equation (4).

The flow chart is described as in Figure 1.

\section{Experiment and analysis}

All of these pictures in this paper are from the internet. In experiments, we applied minimum filtering, and the patch size was set to $9 \times 9$. In Equation (3), $\omega$ was equal to 0.95 . The parameters were $r=40, \varepsilon=0.001$ for the fast guided filter [10]. In order to compare these algorithms, we didn't enhance these results after processing. The program was written in C++ using Microsoft Visual 2008. We installed OpenCV 2.4.1 Library for programming. Our CPU was Intel(R) Core(TM) i5-3450 CPU @3.10GHz. Computer memory was 8G. Operating system was Windows 7.

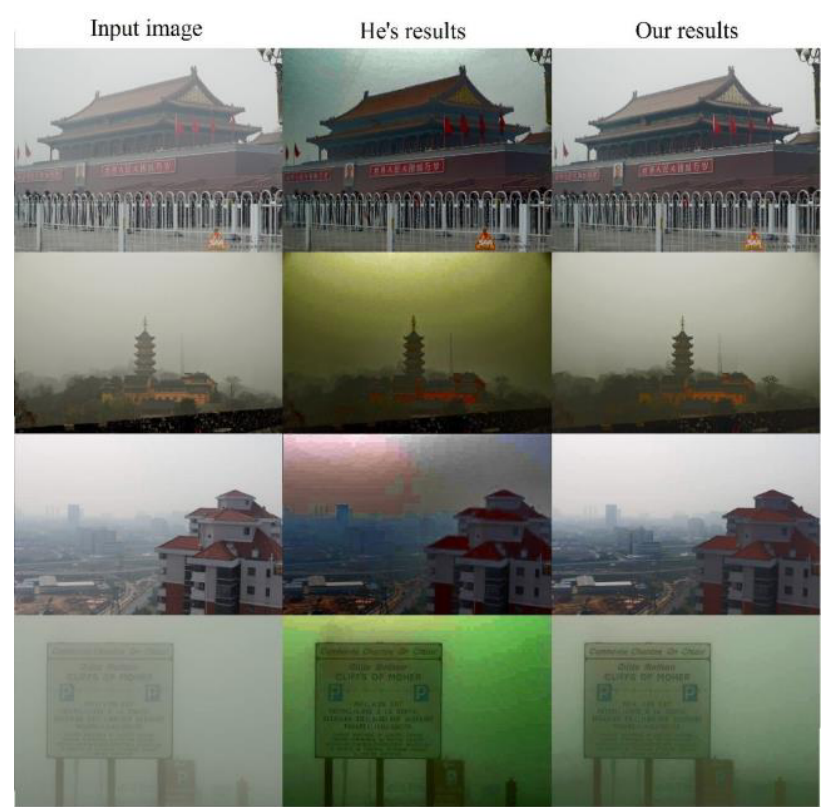

Figure 2. Comparison with He's work [7]. From left to right: Input image, He's results, our results

We selected the four pictures. All pictures both included large-area sky region. In experiments, we compared our method with He's method [7], [10]. These results can be shown in Figure 2 and Table 1. According to Figure 2, we observed that these samples encountered color distortion when there is large-area sky region and utilizing He's method [7], [10]. Only our proposed method can deal with color distortion in all cases.

Table 1. Consuming time.

\begin{tabular}{|l|l|l|l|c|}
\hline $\begin{array}{c}\text { Sample } \\
\text { Number }\end{array}$ & $\begin{array}{c}\text { He's } \\
\text { method by } \\
\text { Guider } \\
\text { filter(ms) }\end{array}$ & $\begin{array}{c}\text { Our } \\
\text { method by } \\
\text { Guider } \\
\text { filter(m) }\end{array}$ & $\begin{array}{l}\text { Our } \\
\text { method by }\end{array}$ & $\begin{array}{c}\text { Picture } \\
\text { size } \\
\text { (pixels) } \\
\text { Guider } \\
\text { filter(ms) }\end{array}$ \\
1 & 733 & 1451 & 406 & $690 \times 517$ \\
2 & 172 & 328 & 93 & $320 \times 240$ \\
3 & 359 & 687 & 187 & $500 \times 333$ \\
4 & 515 & 967 & 266 & $585 \times 413$ \\
\hline
\end{tabular}

In Table 1, we gave the time consumption comparison with He et al. [7], [10]. As can be seen, our approach will spend more time if the transmission map was refined by guider filtering [9]; but, aided by fast guider filtering [10], our approach is much faster than others and may achieve the real-time requirement.

\section{Conclusion}

In the paper, we presented an improved method based on He's work [7], [10] to deal with color distortion. The method firstly determined the inverse image and corresponding dark channel images, and then applied minimum filtering. Next, we calculated the transmission maps and refined it by the fast guider filter [10]. Then, we modified original transmission map based on it. The final transmission map can be used to obtain haze-free image. In final, we can deal with color distortion and enhance the visibility. In future work, we will extend our work to real-time video dehazing.

\section{Acknowledgment}

We thank He Kaiming for generating the results of his method [7], [10]. The authors were supported by the Shinan District Science and technology development fund of Qingdao City under Grant No. 2014-14-013-ZH and Qingdao pioneering and innovative leading talent project under Grant No. 13-CX-23.

\section{References}

1. S. G. Narasimhan, S. K. Nayar, Contrast restoration of weather degraded images. IEEE Transactions on pattern Analysis and Machine Intelligence (PAMI). 25:713-724 (2003)

2. Y. Y. Schechner, S. G. Narasimhan, and S. K. Nayar, Instant dehazing of images using polarization. Processing of 2006 IEEE Conference on Computer Vision and Pattern Recognition CVPR. 1:325 (2006)

3. Kopf, J., Neubert, B., Chen, B., Cohen, M., CohenOr, D. et al., Deep photo: Model-based photograph enhancement and viewing. Processing of the 1st ACM SIGGRAPH Conference and Exhibition on Computer Graphics and Interactive Techniques in Asia. 27(5):110.1-110.6 (2008) 
4. Tan R T., Visibility in bad weather from a single image. Processing of 2008 IEEE Conference on Computer Vision and Pattern Recognition (CVPR). 1:1-8 (2008)

5. Fattal R, Single image dehazing. Processing of the 1st ACM SIGGRAPH Conference and Exhibition on Computer Graphics and Interactive Techniques in Asia. 27(3):1-9 (2008)

6. Jin-Hwan Kim, Won-Dong Jang, Jae-Young Sim., O Chang-Su Kim: Optimized contrast enhancement for real-time image and video dehazing. Journal of Visual Communications and Image Representation. 24(3): 410-426 (2013)

7. He Kaiming, Sun Jian, Tang Xiaoou, Single image haze removal using dark channel prior. Processing of 2009 IEEE Conference on Computer Vision and Pattern Recognition (CVPR). 1956-1963 (2009)

8. Levin A, Lischinski D, Weiss Y, A closed form solution to natural image matting. Processing of 2006 IEEE Conference on Computer Vision and Pattern Recognition (CVPR). 1: 61-68 (2006)

9. He Kaiming, Sun Jian, Tang Xiaoou, Guided Image Filtering. IEEE Transactions on Pattern Analysis and Machine Intelligence. 35(6):1397-1409 (2013)

10. He Kaiming, Sun Jian, Fast Guided Filter. http://arxiv.org/abs/1505.00996. (2015)
11. Jiang Jianguo, Hou Tianfeng, Qi Meibin, Improved algorithm on image haze removal using dark channel prior. Journal of Circuits and Systems. 16(2):7-12 (2011)

12. Sun Xiaoming, Sun Junxi, Zhao Lirong, Cao Yonggang, Improved algorithm for single image haze removing using dark channel prior. Journal of Image and Graphics, 19(3):381-385 (2014)

13. Wang Gangyi, Ren Guanghui, Jiang Lihui, Quan Taifan, Single image dehazing Algorithms based on sky region segmentation, Information Technology Journal. 12( 6):1168-1175 (2013)

14. Deng Yue, Wang Yanjie, Li Jing-yu, Chen Xiaoxi, Zhang Ye, Improvement of enhancement algorithm for aerial image. Laser \& Infrared. 42(9):1080-1085 (2012)

15. Ancuti C O, Ancuti C, Hermans C, et a1, A Fast Semi inverse Approach to Detect and Remove the Haze from a Single Image. 10th Asian Conference on Computer Vision (ACCV 2010). Part II. LNCS, 6493, pp. 501-514 (2014)

16. Tang Ketan, Yang Jianchao, Wang Jue, Investigating Haze-relevant Features in A Learning Framework for Image Dehazing. IEEE Conference on Computer Vision and Pattern Recognition (CVPR). 2995-3002 (2014) 